\title{
ADVANGES IN ANTIMITOTICS
}




\section{Introduction au Symposium par le Prof. L. Gedda}

Je souhaite la bienvenue, et j'adresse mes remerciements, à tous les chercheurs et savants, venus de diverses régions d'Europe et d'Amérique et qui, aujourd'hui, se trouvent réunis dans notre Institut pour discuter des problèmes qui ne peuvent pas être plus actuels, concernant les recherches sur les antimitotiques.

Je remercie les Autorités qui ont bien voulu donner leur adhésion à ce Symposium et ont voulu l'honorer de leur présence.

Notre réunion prend une signification particulière du fait que les études sur les antimitotiques à régard de la chimiothérapie expérimentale des tumeurs ne remontent pratiquement, de façon officielle, qu'à une vingtaine d'années. C'est en effet en 1946 que parurent les travaux de Haddow et Sexton sur "Influence of carbamic esters (uretanes) on experimental animal tumors » (Nature, I57, I946) et ceux de Gilman et Philips sur "The biological actions and the therapeutic applications of the o-chloroethyl-amines and sulphides "(Science, I03, I946). Ce n'est que deux années plus tard que paraîtront les observations de Sidney Farber et de ses collaborateurs sur l'emploi des antifoliques dans la leucémie aiguë des enfants. Au cours des années successives, nous assistons à une floraison de recherches sur les antimitotiques, spécialement à Londres, au Chester Beatty, à New York, au Sloan-Kettering, et à Boston, au Children's Cancer Research Foundation. Je ne rappellerai que les étapes les plus importantes de ces recherches qui ont conduit à une sélection de substances antimitotiques d'une efficace action antitumorale: en 1953, apparaissent la 6-mercaptopurine, le Colcemid, le dyméthansulphoniloxibutane; en I956, le Chlorambucil; en 196o, le Trenimon, l'Endoxan, la Vinblastine, le 5-Fluorouracil; en I962, la Vincristine et l'hydroxyurée; en 1963 , les méthylidrazines. Et ce ne sont là que quelques uns des nombreux cytostatiques étudiés jusqu'à présent.

Les antimitotiques revêtent un grand intérêt du point de vue cytologique, génétique et clinique. L'étude des diverses classes de substances antimitotiques a permis d'étudier divers aspects de la dynamique cellulaire. A cet égard, ont été d'une utilité particulière les agents qui appartiennent au groupe des antimétabolites, comme les antipurines et les antipyrimidines.

Certains antimitotiques, comme les agents stathmocinétiques et en particulier les composés cholchiciniques et la Vinblastine, ont été employés par divers Auteurs comme «tools » pour l'étude de la prolifération cellulaire.

Il m'est particulièrement agréable de mettre l'accent sur l'importance des antimitotiques dans le domaine de la génétique. On peut bien dire qu'avec les antimi- 
totiques s'est ouverte l'ère des recherches sur les mutagènes chimiques. Rapoport, en 1946, relate l'effet mutagénique de la formaline dans la Drosophile, mais, dès I942, des résultats positifs sur l'action mutagène des agents chimiques avaient déjà été obtenus par Auerbach et Robson avec l'yprite. Cause la guerre, ces résultats avaient été gardés secrets et n'ont été divulgués qu'en 1946. Des recherches successives amèneront Auerbach et Robson à démontrer une remarquable action mutagène, comparable à celle des rayons $\mathrm{X}$, dans les yprites azotées. Ensuite, ces composés furent expérimentés et appliqués dans la thérapie des tumeurs.

Avant les recherches de Auerbach et Robson, de nombreuses tentatives avaient été faites pour provoquer des mutations avec des substances chimiques, mais les résultats obtenus n'avaient pas fourni des preuves définitives à l'égard de l'existence d'une mutagénèse chimique. Après la découverte de l'action mutagène des yprites azotées beaucoup d'autres substances mutagènes ont été décrites, bon nombre d'entre elles appartenant justement au groupe des antimitotiques.

Notre Institut s'intéresse beaucoup au problème des antimitotiques. Les recherches faites sur ces substances sont parmi les principaux arguments d'étude du Centre Hématologique de cet Institut, dirigé par le Prof. Cardinali et Mme Cardinali.

Si j'ai souligné le rôle des antimitotiques dans le domaine de la génétique, je ne peux terminer et conclure cette brève salutation sans rappeler l'importance que ces substances assument dans la chimiothérapie expérimentale des tumeurs ainsi que dans le traitement des diverses néoplasies humaines. Pour ne citer qu'un exemple, je rappellerai l'effet des antifoliques, de la 6-mercaptopurine et de la Vincristine dans les leucémies aiguës. Grâce à l'emploi de ces substances, utilisées seules ou en thérapies associées, la survivance dans les leucémies aiguës, surtout chez les enfants, est de nos jours prolongée de beaucoup. Serait-il le seul, que ce fait suffirait à nous aiguillonner pour développer et étendre les recherches sur les antimitotiques. Je souhaite donc que les discussions de cette réunion soient fructueuses, et qu'elles servent de stimulant à chacun de nous pour un plus efficace travail de recherche alors que nous regagnerons, à la clôture de ce Symposium, nos laboratoires et nos cliniques. 